\title{
High-speed microstrip multi-anode multichannel plate detector system
}

Andreas Riedo, Marek Tulej, Urs Rohner, and Peter Wurz

Citation: Review of Scientific Instruments 88, 045114 (2017); doi: 10.1063/1.4981813

View online: https://doi.org/10.1063/1.4981813

View Table of Contents: http://aip.scitation.org/toc/rsi/88/4

Published by the American Institute of Physics

\section{Articles you may be interested in}

Absolute optical responsivity down to the photon counting level with a photomultiplier tube

Review of Scientific Instruments 88, 043104 (2017); 10.1063/1.4979812

New Products

Review of Scientific Instruments 88, 049501 (2017); 10.1063/1.4979931

Sputtering of pure boron using a magnetron without a radio-frequency supply

Review of Scientific Instruments 88, 043506 (2017); 10.1063/1.4980149

Development of a bio-magnetic measurement system and sensor configuration analysis for rats

Review of Scientific Instruments 88, 044704 (2017); 10.1063/1.4979071

Calibration and characterization of a highly efficient spectrometer in von Hamos geometry for 7-10 keV xrays

Review of Scientific Instruments 88, 043110 (2017); 10.1063/1.4981793

Note: Manganin foil sensor for small uniaxial stress

Review of Scientific Instruments 88, 046106 (2017); 10.1063/1.4981247

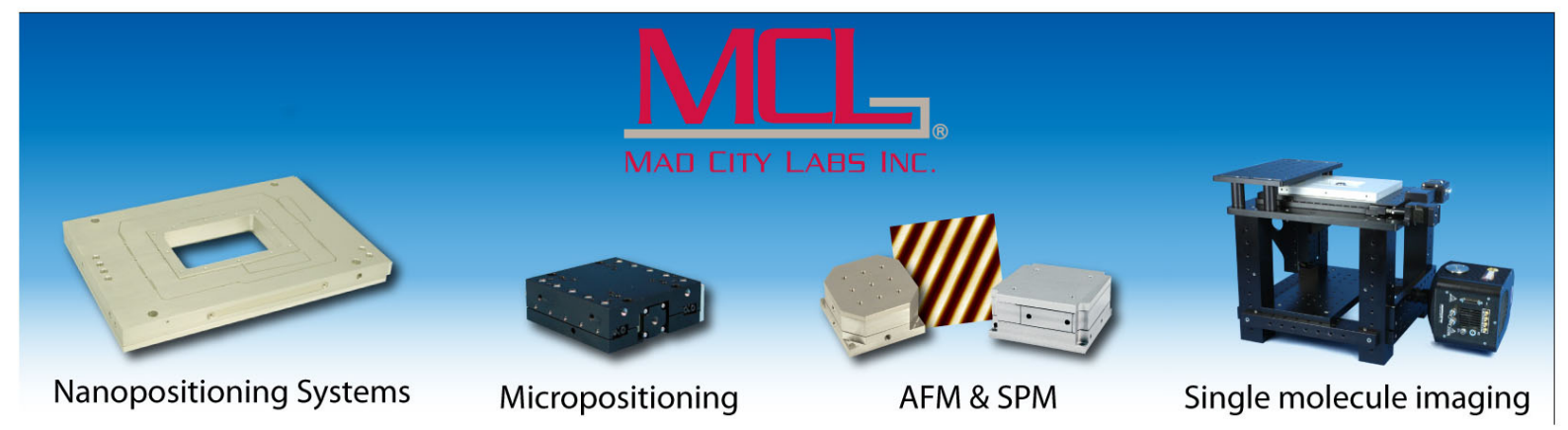




\title{
High-speed microstrip multi-anode multichannel plate detector system
}

\author{
Andreas Riedo, ${ }^{1,2, a)}$ Marek Tulej, ${ }^{2}$ Urs Rohner, ${ }^{2, b)}$ and Peter Wurz ${ }^{2}$ \\ ${ }^{1}$ Leiden Observatory, Sackler Laboratory for Astrophysics, Leiden University, Leiden, The Netherlands \\ ${ }^{2}$ Physics Institute, Space Research and Planetary Sciences, University of Bern, Bern, Switzerland
}

(Received 2 January 2017; accepted 8 April 2017; published online 26 April 2017)

\begin{abstract}
High-speed detector systems with high dynamic range and pulse width characteristics in the subnanosecond regime are mandatory for high resolution and highly sensitive time-of-flight mass spectrometers. Typically, for a reasonable detector area, an impedance-matched anode design is necessary to transmit the registered signal fast and distortion-free from the anode to the signal acquisition system. In this report, a high-speed microstrip multi-anode multichannel plate detector is presented and discussed. The anode consists of four separate active concentric anode segments allowing a simultaneous readout of signal with a dynamic range of about eight orders of magnitude. The impedance matched anode segments show pulse width of about $250 \mathrm{ps,} \mathrm{measured} \mathrm{at} \mathrm{full} \mathrm{width} \mathrm{at} \mathrm{half} \mathrm{maximum,}$ and rise time of $\sim 170 \mathrm{ps}$, measured with an oscilloscope with a sampling rate of $20 \mathrm{GS} / \mathrm{s}$ and 4 $\mathrm{GHz}$ analogue bandwidth. The usage of multichannel plates as signal amplifier allowed the design of a lightweight, low power consuming, and compact detector system, suitable, e.g., for the integration into space instrumentation or portable systems where size, weight, and power consumption are limited parameters. Published by AIP Publishing. [http://dx.doi.org/10.1063/1.4981813]
\end{abstract}

\section{INTRODUCTION}

High-speed detector systems are key elements and mandatory for high mass resolution time-of-flight (TOF) mass spectrometry. Slow detector response times (rise/fall times) limit the mass resolution of a TOF mass spectrometer (because $m / \Delta m=t / 2 \Delta t$, with $m$ the mass and $t$ the flight time of an ion) and therefore the overall analytical performance of the system. For TOF mass spectrometry, response times in the subnanosecond regime are typically required for the detection of pulse widths in the nanosecond range. Detector systems based on multichannel plates (MCPs) are usually used in such instrumentation due to their fast response times and high amplification of incoming signal. To date, several different designs of fast MCP detectors are commercially available from commercial suppliers, such as Photonis or Hammamatsu Photonics. The realization of fast response times and the distortion free transmission of recorded signals is highly demanding and special attention needs to be devoted on the electronic design of the detection system, e.g., impedance matched anode design, the integration of dedicated signal lines and couplings, among others. The concept of an impedance matched anode design is known since 1970s and was first introduced by Wiza by discussing a conical anode design. ${ }^{1}$

Since decades, we have developed various MCP detector systems for different applications. ${ }^{2-13}$ Most of them were designed for the integration into space instrumentation, including the detector system for the reflectron TOF (RTOF) mass spectrometer onboard European Space Agency's (ESA) Rosetta spacecraft to the comet

\footnotetext{
a) Author to whom correspondence should be addressed. Electronic mail: riedo@strw.leidenuniv.nl

b) Present address: TOFWERK AG, Uttigenstrasse 22, 3600 Thun, Switzerland.
}

67P/Churyumov-Gerasimenko, ${ }^{7}$ for the neutral gas mass spectrometer for the Polar-Balloon Atmospheric Composition Experiment (P-BACE) mission, ${ }^{9,10}$ for the miniature laser mass spectrometer designed for the planetary rover onboard ESA's BepiColombo space mission to Mercury, ${ }^{12}$ or for the gas chromatograph-mass spectrometer for the upcoming Russian Luna-Resurs mission to the Moon. ${ }^{8}$ Typically, strict requirements concerning mass, size, and power consumption need to be fulfilled for an instrument to be part of the scientific payload of a spacecraft. The design of detector systems based on a MCP concept is of high interest in this field of research due to their low power consumption, compact dimensions, light weight, and large active area for detection.

Here we present the design and performance evaluation of a high-speed microstrip multi-anode MCP detector system integrated in our laser ablation/ionization mass spectrometer. ${ }^{14-16}$ The design is based on the detector system developed for the laser mass spectrometer instrument for the lander platform onboard ESA's BepiColombo space mission but significantly and steadily improved since its first version. ${ }^{11}$ Please note that the lander platform was cancelled by ESA due to budgetary reasons and with it all instrumentation foreseen for the lander and rover. The detector system consists of four $50 \Omega$ impedance-matched active anode channels, shows a full width measured at half maximum (FWHM) of recorded signal in the range of $200-300 \mathrm{ps}$, and a rise time of about 170 ps. Furthermore, the simultaneous readout of the electric signals of the active anode channels provide a dynamic range of about eight orders of magnitude as discussed first time by Riedo et al., $2013 .{ }^{17}$

\section{DETECTOR DESIGN}

The described detector system is integrated in a miniature reflectron-type TOF laser ablation/ionization mass spectrometer $(160 \mathrm{~mm} \times \varnothing 60 \mathrm{~mm}$, instrument name LMS $)$ for the 
quantitative and sensitive in situ identification of the elemental and isotope composition of solids and soils on the surface of planetary objects, including asteroids, moons, and planets. The design and principles of operation of LMS, including its measurement performance, are described extensively in previous publications. ${ }^{16-26}$ LMS has the capabilities to provide sensitive and quantitative elemental analysis at the 10 ppb level, ${ }^{16,17}$ accurate isotope ratio measurements, ${ }^{21} 2 \mathrm{D}^{19,20}$ and $3 \mathrm{D}^{26}$ elemental imaging of heterogeneous materials, high resolution chemical depth profiling at sub-nm scale, ${ }^{18,24}$ and most recently demonstrated measurement capabilities towards molecular studies at minimized fragmentation. ${ }^{25}$ Nevertheless, a brief description is provided in the following for a better understanding of the design and functionality of the instrument.

A pulsed laser system is used as an ablation/ionization source of solid sample material. The laser pulses are guided with a laser optical system to the top of the mass spectrometer, which is located within a vacuum chamber, and are focused with a lens system through the center of the ion optical system of the mass spectrometer towards the solid sample material. ${ }^{16}$ Each laser shot is removing and ionizing simultaneously the sample material. Only positively charged species can enter the ion optical system of the mass spectrometer. After entering the system, the ion optical system accelerates (voltage of up to about $-2.5 \mathrm{kV}$ ), focuses (voltage of up to about $-2.5 \mathrm{kV}$ ), and confines the ions towards the field free drift path (drift tube, mass to charge $\mathrm{m} / \mathrm{z}$ separation, voltage of up to about $-1.2 \mathrm{kV}$ ) by passing first the concentric detector system (center hole of $\varnothing 6.4 \mathrm{~mm}$ ). The ions are subsequently reflected at the ion mirror (voltages in the range of about $-200 \mathrm{~V}$ to $+500 \mathrm{~V}$ ), pass a second time the field free drift path, and are finally registered by the detector system (Fig. 1). Depending on the measurement campaign, including, e.g., element analysis, isotope analysis, chemical depth profiling, and characteristics of sample material, the applied voltages on the ion optics vary. Because of the small size of the LMS instrument, the varying plasma conditions for the different samples translate into different ion optical settings. The voltages are optimized for, e.g., best ion transmission of a certain element/isotope or mass resolution $(\mathrm{m} / \Delta \mathrm{m})$ using an adaptive swarm algorithm software. ${ }^{22}$ In Riedo et al., $2013,{ }^{17}$ a complete and representative set of applied voltages is listed.

Ions of different mass are arriving at the detector system in time sequences, according to their $\mathrm{m} / \mathrm{z}$ ratio (according to the TOF measurement principle ${ }^{17}$ ). Typically, a full TOF spectrum, ranging from the detection of hydrogen to lead, has a time length of about $13 \mu \mathrm{s}$ in the LMS instrument, which corresponds to the arrival time of lead. ${ }^{17}$ Once the ion cloud of the same $m / z$ ratio arrives at the MCP detector system, the ions are electrically amplified by their initiated electron avalanches. The avalanches are collected finally by the segmented anode system (Fig. 1).

Figure 2 shows the electric schematics of the LMS detector system. The incoming ions are electrically amplified by the MCP detector system, which consists of two matched MCPs in chevron configuration with an active area of $40 \mathrm{~mm}$ in diameter (Photonis, Inc. USA, Long-Life ${ }^{\mathrm{TM}}$ Microchannel

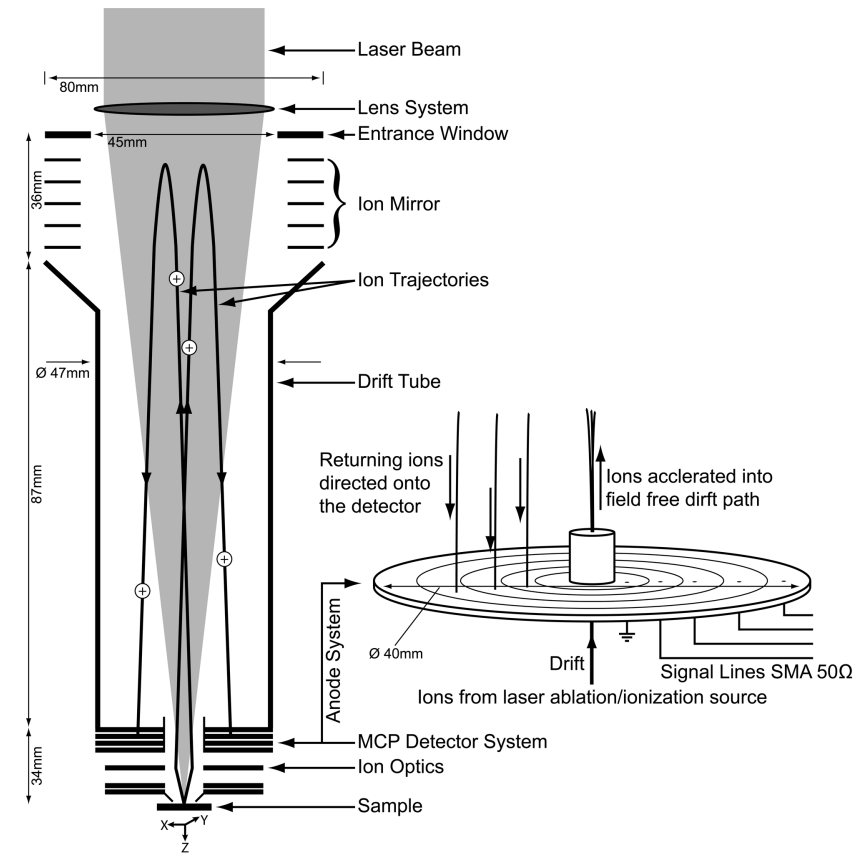

FIG. 1. Left: A schematic drawing of the LMS instrument. Only positively charged ions can enter the mass spectrometer after the ablation and ionization process of sample material. The positive ions are guided with the ion optical system through the detector system, pass the field free drift path (drift tube), and are reflected subsequently at the ion mirror towards the MCP detector system. ${ }^{11,16}$ Right: Side view of the mechanical schematic of the anode system, part of the MCP detector system.

Plate Set, MCP 40/12/8/8 D 60:1 MP 6.4CH EDR, MS; with $\mathrm{D}$ for detection, MP for mounting pad, $\mathrm{CH}$ for center hole in $\mathrm{mm}$, EDR for extended dynamic range, and MS for matched set). A set of dedicated resistors $\left(R_{1}\right.$ and $R_{2}$, in series to the high voltage supplies of the plates) and capacitors ( $\mathrm{C}_{\mathrm{MCP} 1}$ and $\mathrm{C}_{\mathrm{MCP} 2}$, in parallel to the plates) is connected in close proximity to each of the MCPs allowing high speed provision of electrons for the electron pulses after an ion hit on the detector system. The implementation of the capacitors in parallel configuration allows best, in terms of speed, electron feeding of the MCPs. ${ }^{5}$ A segmented microstrip anode plate, a system of 5 concentric areas (channels) each isolated to each other, is used to collect the electron pulses from the registered ions.

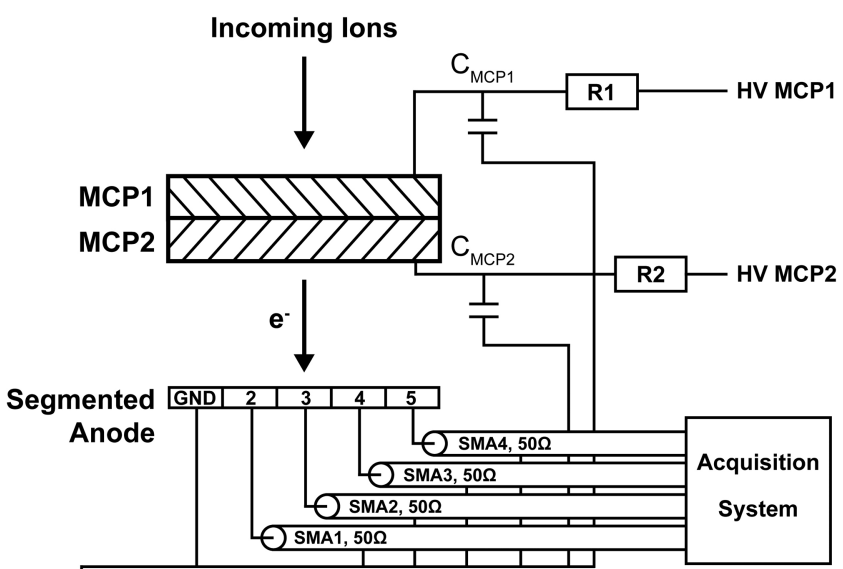

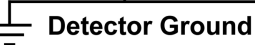

FIG. 2. Electric schematics of the LMS detector system. 
TABLE I. Key parameters of the LMS detector system.

\begin{tabular}{lc}
\hline \hline Overall dimension & $7.5 \mathrm{~mm} \times \emptyset 70 \mathrm{~mm}$ \\
Weight & $100 \mathrm{~g}$ \\
Power consumption & $\leq 1 \mathrm{~W}$ \\
Voltages & \\
MCP 1 & $-1.80 \mathrm{kV}$ to $-2.1 \mathrm{kV}$ \\
MCP 2 & $-250 \mathrm{~V}$ \\
Anode & PCB \\
Design & Teflon \\
Substrate & Au \\
Coating & 5, active 4 \\
\# Channels & Circular ring thickness of $2.8 \mathrm{~mm}$, \\
Channel dimension & spacing $0.2 \mathrm{~mm}$ \\
& 50 impedance matched, \\
Coupling & microstrip design \\
Integrated MCPs & $\varnothing 50 \mathrm{~mm}$ \\
Dimensions & $\emptyset 40 \mathrm{~mm}$ \\
Active area & $\varnothing 8 \mu \mathrm{m}$ \\
Pore size & $8^{\circ}$ \\
Pore angle & $60: 1$ \\
L/D & $12 \mu \mathrm{m}$ \\
Pitch & Photonis, Inc., USA \\
Design & \\
Supplier & \\
\hline \hline
\end{tabular}

The anode is a custom-made high frequency circular microstrip design and is realized on a printed circuit board (PCB). The inner most area is grounded whereas the subsequent four channels (2-5, Fig. 2) are designed for $50 \Omega$ electrical impedance. Vacuum compatible coaxial cables are connected at the segmented anode plate transporting the signals to the high speed analog-to-digital converter (ADC) and data acquisition system by passing first the flange with subminiature version A (SMA) feed-throughs at the UHV chamber. In total, about $1 \mathrm{~m}$ of SMA cable is used between anode channels and acquisition system. The length of the coaxial cables is chosen as short as possible to prevent peak broadening and distortion during signal transmission and to minimize noise pick-up. Special attention has been devoted for the definition of the grounding concept of the detector system by means of a single grounding point for all high voltage connections. In Table I, the key parameters of the LMS detector system are listed.

\section{MEASUREMENTS}

\section{A. Single ion events}

Measurements were conducted within the LMS vacuum chamber, which has a typical base pressure of about $5 \times 10^{-8}$ mbar. ${ }^{8,9}$ Ion registrations on the LMS detector from ionized residual gas inside the vacuum chamber from the vacuum gauge at a rate of about $1 \mathrm{ion} / \mathrm{s}$ were used for the characterization of each anode area of the detector system (2-5, Fig. 2). Typically, two high speed ADC data acquisition cards (Keysight Technologies, each with two active channels, 8 bit, up to $4 \mathrm{GS} / \mathrm{s}$ ) are used to record the electric signals during mass spectrometric measurements. However, for the quantification of the detector performance, a high speed digital storage oscilloscope (DSO, Tektronix, TEK TDS7404) with a sampling rate of $20 \mathrm{GS} / \mathrm{s}$ and an analogue bandwidth of $\mathrm{BW}=4 \mathrm{GHz}$ was used. The sampling speed, corresponding to $50 \mathrm{ps}$ time intervals on the time axis, is sufficiently high but at limit for the characterization of the reported detector system (see Sec. IV A).

Potential differences in the range of $\Delta \mathrm{V}$ is $1550 \mathrm{~V}$ to $1850 \mathrm{~V}$ were applied between the stack of the two MCPs (HV MCP1-HV MCP2 in Fig. 2), by stepwise increasing this potential difference by $50 \mathrm{~V}$. The applied voltage at the exit of the second MCP (HV MCP2, Fig. 2) was kept constant at $-250 \mathrm{~V}$ during the measurements. Home-made high-voltage power supplies, offering sufficient high voltage resolution of the applied voltages $(\sim 1 \mathrm{~V})$, were used for the performance evaluation. For each channel and each applied potential difference, ten single ion events were recorded. The raw data were forwarded from the oscilloscope to a host-computer for subsequent data analyses. Standard fit routines (provided by the program OriginPro, OriginLab) were used for the characterization of recorded electric signals, which is the full width at half maximum (FWHM), and rise time of detected electric signals. The function Gauss Amplitude was used as fit function, showing best match with the detected signal and defined by

$$
\mathrm{y}=\mathrm{y}_{0}+\mathrm{A}^{*} \exp \left(-0.5^{*}\left(\left(\mathrm{x}-\mathrm{x}_{\mathrm{c}}\right) / \mathrm{w}\right)^{2}\right),
$$

where $y_{0}$ denotes the offset (background), $A$ the amplitude, $x_{c}$ the peak center, and $w$ the Gaussian width of the recorded peak.

\section{B. Dynamic range}

Mass spectrometric measurements were conducted on a National Institute of Standard and Technology (NIST) certified reference material (SRM665) to demonstrate the dynamic range performance of the system. A femtosecond laser system (CPA system, Clark-MXR, Inc., Dexter, MI, USA; pulse width $\tau \sim 190 \mathrm{fs}$, wavelength $\lambda=775 \mathrm{~nm}$, laser pulse repetition rate $\leq 1 \mathrm{kHz}$ ) was used in this study for clean ablation and efficient ionization of sample material. Depending on the measurement campaign, ranging from chemical depth profiling analysis of semiconductor materials ${ }^{18,24}$ to chemical analysis of micrometer fossils embedded in a host matrix ${ }^{23}$ the laser irradiance needs to be adjusted, typically ranging from sub $\mathrm{TW} / \mathrm{cm}^{2}$ to tens of $\mathrm{TW} / \mathrm{cm}^{2}$, corresponding to pulse energies in the range of sub $\mu \mathrm{J}$ to a few $\mu \mathrm{J}$ and ablation craters with about 10 to 20 $\mu \mathrm{m}$ in diameter. Here, a laser irradiance of about $0.3 \mathrm{TW} / \mathrm{cm}^{2}$ was applied for ablation and ionization of sample material. The electric signals were recorded using the two high-speed ADC data acquisition cards described above in this contribution. The mass spectrum used to evaluate the dynamic range consists of an accumulation of 200000 single laser shot mass spectra. The single laser shot mass spectra were sequentially accumulated first on the acquisition system to a single mass spectrum consisting of 2000 single laser shot mass spectra, which is stored on the host computer (in total 100 such mass spectra). A software routine written in Matlab was used for post-processing these data and for the co-adding of these 100 mass spectra into the final mass spectrum. 


\section{DISCUSSION}

\section{A. Single ion events}

A typical recording with the DSO of the electric signal of a single ion event is shown in the top panel of Fig. 3 as a black line (anode channel $4, \Delta \mathrm{V}_{\mathrm{MCP}}$ over MCP stack of $-1.65 \mathrm{kV}$ ). The red line in Fig. 3 corresponds to the Gauss Amplitude fit of the measured data, as discussed in the previous paragraph. In the bottom panel, the residuals between the fit and raw data are shown, considering only the time range used for the fit. In this example, a FWHM of 266 ps, a rise time of 190 ps ( $10 \%$ to $90 \%$ level), and a signal amplitude of $-4.73 \mathrm{mV}$ were measured. Considering the peak broadening by the ana$\log$ bandwidth of the oscilloscope, for which the rise time can be estimated as $t_{\text {rise }} \approx 1 /(3 \times \mathrm{BW})=83 \mathrm{ps},{ }^{2,5}$ we calculate a deconvolved rise time of $\sim 170 \mathrm{ps}$. Additionally, the peak shape is highly symmetric, and no significant electrical ringing to the tailing side of the peak is observed. Ringing and broadening are typical results of an impedance mismatch somewhere along the transmission between acquisition and detector system.

Many of today's commercially available standard MCP detector systems (chevron configuration) show typical pulse widths (FWHM) in the order of $500 \mathrm{ps,} \mathrm{rise} \mathrm{times} \mathrm{below} 300$ ps, and consist typically of a single anode, see, e.g., product line of Hammamatsu Photonics or Photonis. The Gen2 UltraFast $^{\mathrm{TM}}$ TOF Detector system from the company Photonis is, to our best knowledge, one of the fastest MCP detector system today commercially available on the market. The system (active area of $18 \mathrm{~mm}$ in diameter, MCP pore diameter of
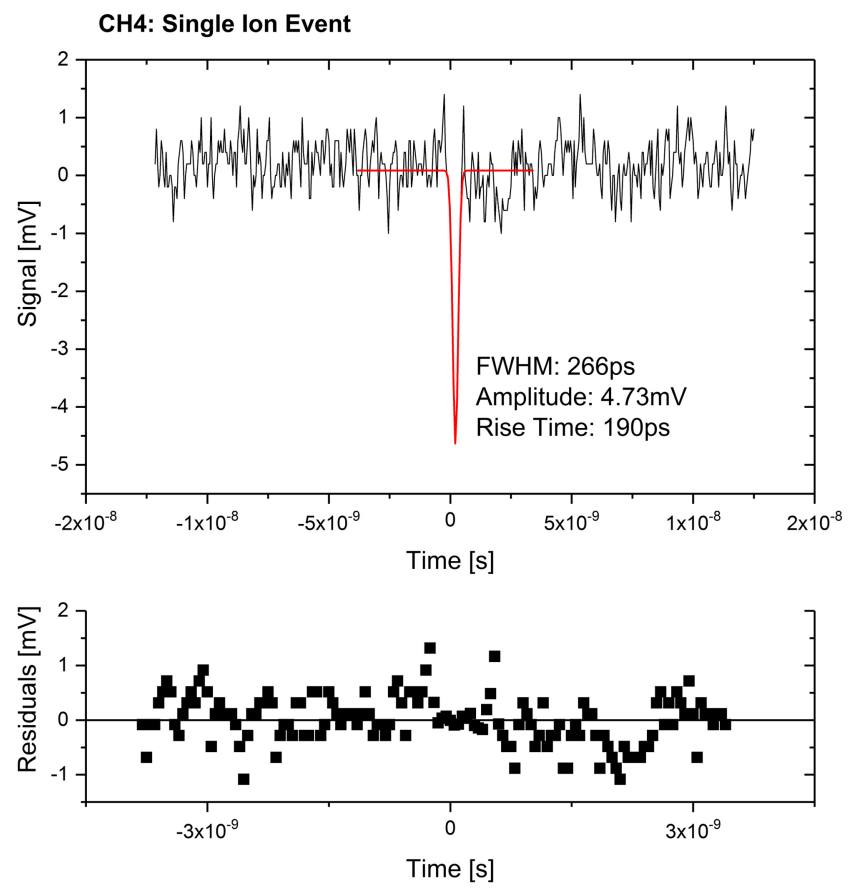

FIG. 3. Single ion event registered on anode channel 4 recorded with the DSO (20 Gs/s, $4 \mathrm{GHz} \mathrm{BW}$ ); a potential difference $\Delta \mathrm{V}_{\mathrm{MCP}}$ of $-1.65 \mathrm{kV}$ was applied over the MCP stack. Top panel: raw signal (black line) with a Gauss Amplitude fit of the pulse (red line) is shown. The detected signal shows a pulse width measured at FWHM of about 266 ps, signal amplitude of about $-4.7 \mathrm{mV}$, and a rise time of $190 \mathrm{ps}$. Bottom panel: residuals between raw and fitted data over time range used for fit (see top panel).
$2 \mu \mathrm{m}$, single anode) shows pulse widths (FWHM) shorter than $200 \mathrm{ps}$ and rise times close to $125 \mathrm{ps}$. The usage of MCPs with pore diameters of only a few microns is of considerable interest if fastest pulse characteristics are required for the experimental setup, since rise time and pulse width roughly scale with the pore size of the channel plates. ${ }^{4,5,27}$

For the large active area of $\varnothing 40 \mathrm{~mm}$ needed for our system, MCPs with pore diameter of $2 \mu \mathrm{m}$ are too thin to be practical. We decided to use multichannel plates with pore diameters of $8 \mu \mathrm{m}$. To cover the active area, a requirement of our instrument setup, and to allow for a large dynamic range, we designed a multi-anode system for simultaneous readout of electrical signals from this large active area. The current design fulfils our target parameters of high amplification of detected ions and high dynamic range of about eight orders of magnitude, discussed in Sec. IV B.

Figure 4 shows the correlation between measured pulse width (FWHM) and signal amplitude of detected single ion signals for all anode channels (2-5, Fig. 2). All channels show a comparable measurement performance in terms of pulse width and signal amplitude dependences on the applied MCP voltage. A logarithmic trend (solid black line) between measured pulse width and signal amplitude can be observed for all channels. The measurements scatter with about $\pm 10 \%$ around this logarithmic trend line. Fastest signals with a measured pulse width of about 200-300 ps (not bandwidth corrected) with lowest signal amplitudes of about $2 \mathrm{mV}$ were measured at lowest applied potential difference $\left(\Delta \mathrm{V}_{\mathrm{MCP}}=-1.55 \mathrm{kV}\right)$. By increasing the applied potential difference at the detector system, an increase of signal amplitudes with increasing pulse width can be observed. We conjecture that space charge effects inside the pores of the MCPs are the source of this broadening effect. At an increased number density of electrons (increased space charge) within a single MCP pore, Coulombic repulsion becomes non-negligible and will spread the electron cloud along the pore axis and hence broadens the registered charge pulse in time.

In Table II, an estimation of generated electrons per MCP pore at two extremes of applied potential differences at the detector system $\left(\Delta \mathrm{V}_{\mathrm{MCP}}\right.$ of $-1.55 \mathrm{kV}$ and $\left.-1.85 \mathrm{kV}\right)$ is given. The calculation is based on measurements conducted on anode channel 3 (measured single ion events) and by estimating that the electron cloud generated in one pore of MCP1 is distributed over 10 single pores on MCP2 (based on geometrical considerations). The total number of electrons (or modal gain, 2nd column in Table II) generated for one single ion event can be derived from the integration over time of the detected signal and knowing that we have a $50 \Omega$ impedance matched detector design. The increase of the potential difference of about $300 \mathrm{~V}$ results in an increase of the modal gain, that is, the number of generated electrons in a single MCP pore, of about two decades. As a rule of thumb, every $50 \mathrm{~V}$ increase of the potential difference increases the modal gain by a factor of about 2 .

\section{B. Dynamic range}

The design of a detector system with a dynamic range (simultaneous readout of large and small signals) covering 
CH2: FWHM vs. Signal

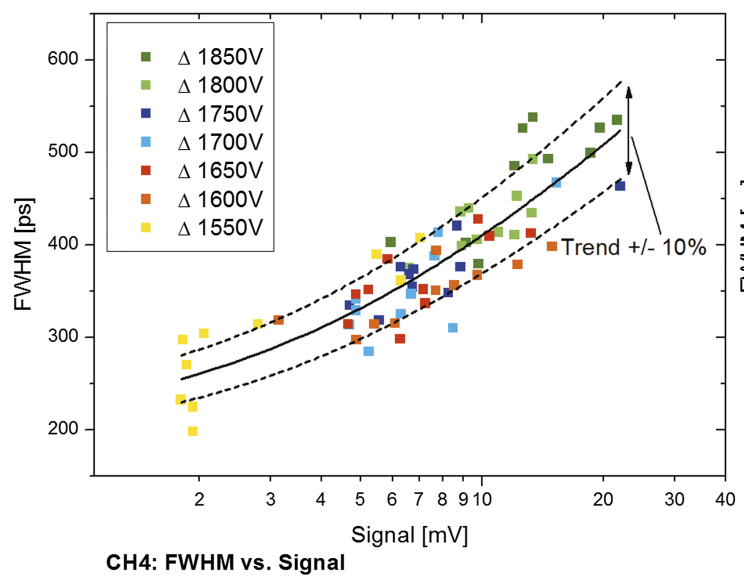

CH4: FWHM vs. Signal

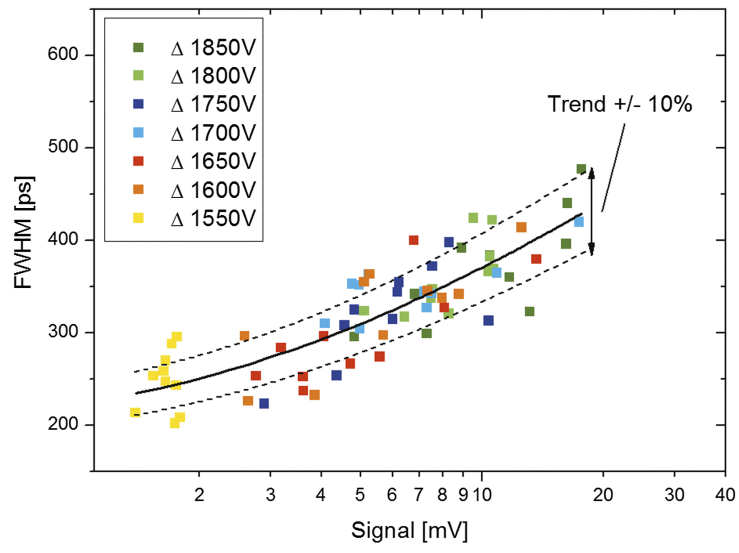

CH3: FWHM vs. Signal

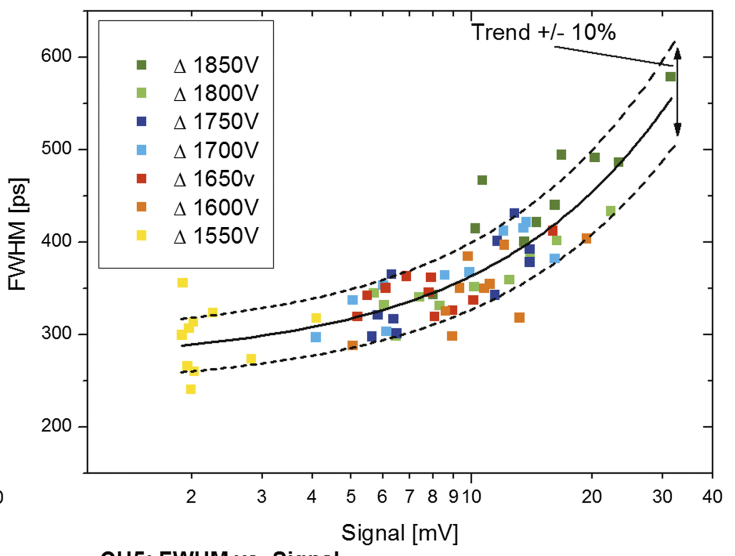

CH5: FWHM vs. Signal

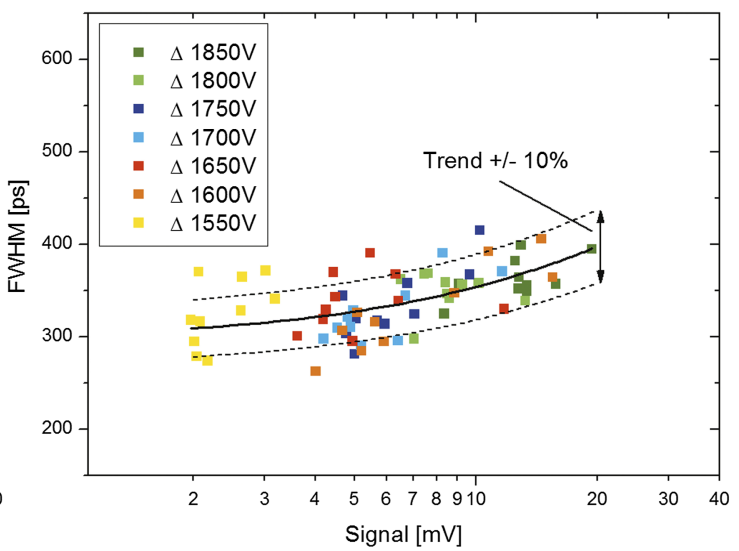

FIG. 4. Correlation between detected signal amplitudes and pulse widths (FWHM) of single ion events measured at anode channels 2-5 and applying potential differences $\Delta \mathrm{V}$ over the MCP stack in the range of $-1.55 \mathrm{kV}$ to $-1.85 \mathrm{kV}$. A logarithmic trend (black solid line) between increasing pulse widths with increasing signal amplitudes for all channels can be observed. The individual measurements scatter around the logarithmic trend line (shown by the dashed curves $\pm 10 \%$ of logarithmic fit).

several orders of magnitude is typical of high interest in mass spectrometric measurements. A high dynamic range allows in single measurements the quantification of the abundance of major to trace elements in a sample or the detection of isotopes of an element showing a high variation in their isotope fractions, e.g., the isotopes of $\mathrm{Pt}$ with variations in isotope abundances from 33.832 for ${ }^{195} \mathrm{Pt}$ to 0.014 for ${ }^{190} \mathrm{Pt}$ (weight percentages). ${ }^{28}$

The segmented anode system of the LMS detector system allows the simultaneous recording and read-out of electric signals at different gain levels because of the different geometric factor of the anodes, the different ion flux from the mass analyser on the anode segments, and the different electronic

TABLE II. Calculation of the modal gain at two extremes of applied potential differences for the estimation of the number of electrons per MCP pore. Measurements conducted on anode channel 3 were used for the calculations. The increase of the potential difference at the detector system by about $300 \mathrm{~V}$ results in an increase of number of electrons of about two decades.

\begin{tabular}{lccc}
\hline \hline $\begin{array}{l}\text { Applied } \\
\Delta(\mathrm{V})\end{array}$ & $\begin{array}{c}\text { \# Electrons } \\
\text { (gain) }\end{array}$ & $\begin{array}{c}\text { \# Electrons/MCP } \\
\text { pore }\end{array}$ & $\begin{array}{c}\text { Measured FWHM/amplitude } \\
(\mathrm{ps} / \mathrm{mV})\end{array}$ \\
\hline 1550 & $\sim 6.4 \times 10^{4}$ & $\sim 6.4 \times 10^{3}$ & $\sim 266 / \sim 2.0$ \\
1850 & $\sim 3.0 \times 10^{6}$ & $\sim 3.0 \times 10^{5}$ & $\sim 580 / \sim 31.4$ \\
\hline \hline
\end{tabular}

gains of the signal acquisition system for each channel. For example, mass spectrometric measurements with LMS are typically accomplished by recording the signals at three different electronic gain levels, ranging from the so called High-Gain (HG), Mid-Gain (MG), and Low-Gain (LG) channel. ${ }^{17}$ In the HG channel, the gain level is set to the highest possible value allowing the detection of the smallest electric signals corresponding to the trace elements or isotopes within the sample material (element and isotope abundances down to ppb level). In this channel intense signals, corresponding to elements of major abundance, are saturated in the electronics and cannot be quantified correctly. These elements are recorded in the LG channel with the lowest gain value. Elements and isotopes of low abundance that are hardly detected in the LG channel or are saturated in the HG channel can be recorded in the MG channel. Once the measurement is accomplished in all channels simultaneously, a superposition of these channels can be realized by means of selecting detected elements/isotopes in channels that are not saturated. The superposition procedure is discussed in more detail in a previous publication where the elements $\mathrm{B}$ and $\mathrm{C}$ are used as elements/isotopes to inter-calibrate between the three active channels. ${ }^{17}$

In Figure 5, the superposition of the LG (in red) and HG (in black) spectrum is shown. For simplicity, only the 


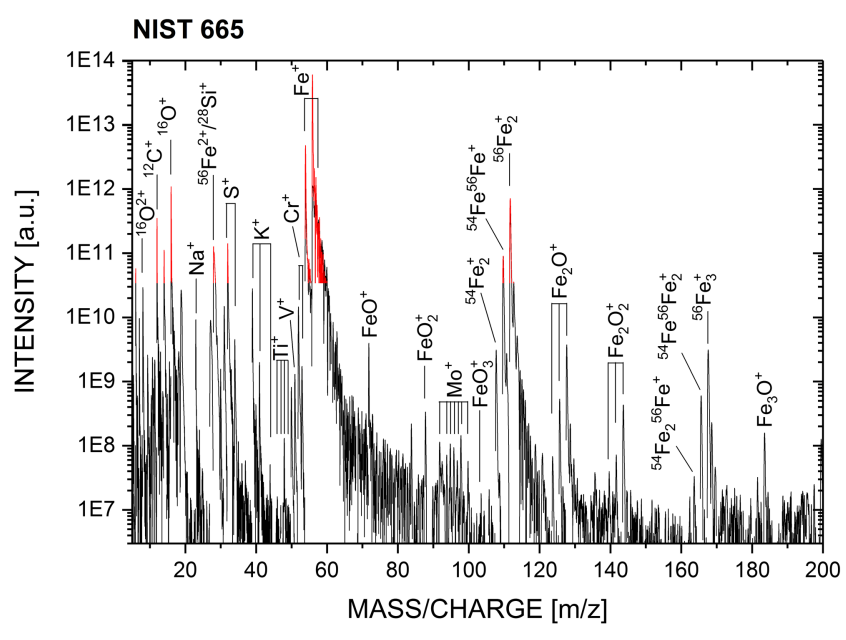

FIG. 5. Superimposed HG (black) and LG (red) mass spectra of a measurement campaign conducted on NIST SRM 665. In this campaign, a dynamic range of about 7.5 orders of magnitude is accomplished.

signals from HG and LG channels are shown. High abundant species are detected in the LG spectrum without artefacts of saturation. In the HG spectrum, low abundant species can be analyzed, e.g., Mo isotopes with abundances of only a few ppm (atomic fraction), however, at the expense that high abundant elements/isotopes are saturated. Taken together, the HG and LG spectra allow for a spectrometric analysis over about 7.5 decades of signal intensities.

\section{CONCLUSIONS}

The design and performance of a microstrip multi-anode multichannel plate detector system originally designed for laser ablation/ionization mass spectrometry are discussed. The system consists of four active impedance matched microstrip concentric anode channels for simultaneous read-out of the electrical signals from the MCPs. The different channels can be recorded at different gain levels, which allow mass spectrometric investigations with a dynamic range of about eight orders of magnitude. The implemented electrical concept, including the dedicated sets of resistors and capacitors, allows fast rise times of about 170 ps (bandwidth corrected) and measured pulse widths in the range of 200-300 ps of recorded single ion events. The compact, lightweight, and low power consuming design of the detector system allows the implementation into space instrumentation or portable instruments where these parameters are of special interest.

\section{ACKNOWLEDGMENTS}

This work is supported by the Swiss National Science Foundation.

${ }^{1}$ J. L. Wiza, Nucl. Instrum. Methods 162, 587 (1979).

${ }^{2}$ R. Schletti, P. Wurz, S. Scherer, and O. H. Siegmund, Rev. Sci. Instrum. 72(3), 1634 (2001).

${ }^{3}$ P. Wurz and R. Schletti, Rev. Sci. Instrum. 72(8), 3225 (2001).

${ }^{4}$ P. Wurz and L. Gubler, Rev. Sci. Instrum. 65(4), 871 (1994).

${ }^{5}$ P. Wurz and L. Gubler, Rev. Sci. Instrum. 67(5), 1790 (1996).

${ }^{6}$ R. Rispoli, E. De Angelis, L. Colasanti, N. Vertolli, S. Orsini, J. A. Scheer, A. Mura, A. Milillo, P. Wurz, S. Selci, A. M. Di Lellis, R. Leoni, M. D'Alessandro, F. Mattioli, and S. Cibella, Opt. Eng. 52(5), 051206 (2013).

${ }^{7}$ O. H. W. Siegmund, K. E. Kromer, P. Wurz, R. Schletti, and H. Cottard, Proc. SPIE 4140, X-Ray and Gamma-Ray Instrumentation for Astronomy XI, 229 (2000).

${ }^{8}$ L. Hofer, P. Wurz, A. Buch, M. Cabane, P. Coll, D. Coscia, M. Gersimov, D. Lasi, A. Sapgir, C. Szopa, and M. Tulej, Planet. Space Sci. 111, 126 (2015).

${ }^{9}$ D. Abplanalp, P. Wurz, L. Huber, I. Leya, E. Kopp, U. Rohner, M. Wieser, L. Kalla, and S. Barabash, Adv. Space Res. 44, 870 (2009).

${ }^{10}$ P. Wurz, D. Aplanalp, M. Tulej, and H. Lammer, Planet. Space Sci. 74, 264 (2012).

${ }^{11}$ U. Rohner, J. Whitby, and P. Wurz, Meas. Sci. Technol. 14, 2159 (2003).

${ }^{12}$ U. Rohner, J. A. Whitby, P. Wurz, and S. Barabash, Rev. Sci. Instrum. 75, 1314 (2004).

${ }^{13}$ M. Tulej, S. Meyer, M. Lüthi, D. Lasi, A. Galli, L. Desorgher, W. Hajdas, S. Karlsson, L. Kalla, and P. Wurz, Rev. Sci. Instrum. 86, 083310 (2015).

${ }^{14}$ D. D. Grieg and H. F. Engelmann, Proc. IRE 40, 1644 (1952).

${ }^{15}$ R. Garg, I. Bahl, and M. Bozzi, Microstrip Lines and Slotlines, 3rd ed. (Artech House, Boston, London, 2013).

${ }^{16}$ A. Riedo, M. Neuland, S. Meyer, M. Tulej, and P. Wurz, J. Anal. At. Spectrom. 28, 1256 (2013).

${ }^{17}$ A. Riedo, A. Bieler, M. Neuland, M. Tulej, and P. Wurz, J. Mass Spectrom. 48, 1 (2013).

${ }^{18}$ V. Grimaudo, P. Moreno-García, A. Riedo, M. B. Neuland, M. Tulej, P. Broekmann, and P. Wurz, Anal. Chem. 87, 2037 (2015).

${ }^{19}$ M. Tulej, A. Riedo, M. B. Neuland, S. Meyer, P. Wurz, N. Thomas, V. Grimaudo, P. Moreno-García, P. Broekmann, A. Neubeck, and M. Ivarsson, Geostand. Geoanal. Res. 38, 441 (2014).

${ }^{20}$ M. B. Neuland, S. Meyer, K. Mezger, A. Riedo, M. Tulej, and P. Wurz, Planet. Space Sci. 101, 196 (2014).

${ }^{21}$ A. Riedo, S. Meyer, B. Heredia, M. B. Neuland, A. Bieler, M. Tulej, I. Leya, M. Iakovleva, K. Mezger, and P. Wurz, Planet. Space Sci. 87, 1 (2013).

${ }^{22}$ A. Bieler, K. Altwegg, L. Hofer, A. Jäckel, A. Riedo, T. Sémon, P. Wahlström, and P. Wurz, J. Mass Spectrom. 46, 1143 (2011).

${ }^{23}$ M. Tulej, A. Neubeck, M. Ivarsson, A. Riedo, M. Neuland, S. Meyer, and P. Wurz, Astrobiology 15, 669 (2015).

${ }^{24}$ A. Riedo, V. Grimaudo, P. Moreno-García, M. B. Neuland, M. Tulej, P. Wurz, and P. Broekmann, J. Anal. At. Spectrom. 30, 2371 (2015).

${ }^{25}$ P. Moreno-García, V. Grimaudo, A. Riedo, M. Tulej, M. B. Neuland, P. Wurz, and P. Broekmann, Electrochim. Acta 199, 394 (2016).

${ }^{26}$ V. Grimaudo, P. Moreno-Garcia, A. Riedo, S. Meyer, M. Tulej, M. B. Neuland, M. Mohos, C. Gütz, S. R. Waldvogel, P. Wurz, and P. Broekmann, Anal. Chem. 89, 1632 (2017).

${ }^{27}$ H. Kume, K. Koyama, K. Nakatsugawa, S. Suzuki, and D. Fatlowitz, Appl. Opt. 27, 1170 (1988).

${ }^{28}$ J. S. Becker, Inorganic Mass Spectrometry-Principles and Applications (John Wiley \& Sons, Ltd., England, 2007). 